\title{
Erratum to: Implantable neurotechnologies: bidirectional neural interfaces-applications and VLSI circuit implementations
}

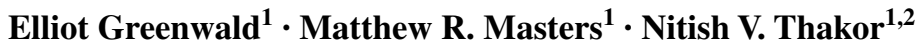

Published online: 29 February 2016

(C) International Federation for Medical and Biological Engineering 2016

\section{Erratum to: Med Biol Eng Comput DOI 10.1007/s11517-015-1429-x}

The original article was published with the following errors.

In the introduction section, at the end of the final paragraph, the following sentences were missed.

"This review is complemented by three companion papers with emphases on electrodes [103], integrated circuit amplifiers [92], and stimulators [89]. Together, they highlight milestones and challenges in the field of implantable neuroprostheses."

Reference 46 was incorrect. The correct reference is given below:

Fetz EE (2015) Chapter 12-restoring motor function with bidirectional neural interfaces. In: Dancause $\mathrm{N}$, Nadeau $\mathrm{S}$, Rossignol S (eds) Sensorimotor rehabilitation at the crossroads of basic and clinical sciences, vol 218 of progress in brain research. Elsevier, Amsterdam, pp 241-252

The online version of the original article can be found under doi:10.1007/s11517-015-1429-x.

Elliot Greenwald

egreenw3@jhu.edu

Nitish V. Thakor

nthakor@jhu.edu; sinapsedirector@gmail.com

1 Department of Biomedical Engineering, Johns Hopkins University, Baltimore, MD, USA

2 Singapore Institute for Neurotechnology (SINAPSE), National University of Singapore, Singapore, Singapore
Reference 70 was incorrect. Hence, it should be removed and the References 71-88 should be renumbered as 70-87.

In Reference 81 (now it is Ref. 80), the journal name was incorrect. The correct journal name should read as "Front Human Neurosci". The correct reference is given below:

Liew S, Santarnecchi E, Buch E, Cohen L (2014) Noninvasive brain stimulation in neurorehabilitation: Local and distant effects for motor recovery. Front Human Neurosci $8: 378$

The following new reference should be inserted as Reference 88:

Muller R, Gambini S, Rabaey J (2012) A $0.013 \mathrm{~mm}^{2} 5 \mu \mathrm{W}$ DC-coupled neural signal acquisition IC with $0.5 \mathrm{~V}$ supply. IEEE J Solid-State Circuits 47(1):232-243

The following new reference should be inserted as Reference 93:

Nishimura Y, Perlmutter SI, Eaton RW, Fetz EE (2013) Spike-timing-dependent plasticity in primate corticospinal connections induced during free behavior. Neuron 80(5):1301-1309

Reference 93-101 should be renumbered as 94-102. Reference 102 should be listed as Reference 104.

The following new reference should be inserted as Reference 105:

Plow EB, Carey JR, Nudo RJ, Pascual-Leone A (2009) Invasive cortical stimulation to promote 
recovery of function after stroke a critical appraisal. Stroke 40(5):1926-1931

Reference 104-140 should be renumbered as 106-142. The reference citations in the text should also be changed accordingly.

All the above-mentioned changes have been corrected in the original article.

The amended list of references $70-142$ is as follows:

\section{References}

70. Kühn AA, Kempf F, Brücke C, Doyle LG, Martinez-Torres I, Pogosyan A, Trottenberg T, Kupsch A, Schneider GH, Hariz MI et al (2008) High-frequency stimulation of the subthalamic nucleus suppresses oscillatory $\beta$ activity in patients with Parkinson's disease in parallel with improvement in motor performance. J Neurosci 28(24):6165-6173

71. Kühn AA, Kupsch A, Schneider GH, Brown P (2006) Reduction in subthalamic $8-35 \mathrm{hz}$ oscillatory activity correlates with clinical improvement in Parkinson's disease. Eur J Neurosci 23(7):1956-1960

72. Kumar K, Taylor RS, Jacques L, Eldabe S, Meglio M, Molet J, Thomson S, Callaghan OJ, Eisenberg E, Milbouw G et al (2007) Spinal cord stimulation versus conventional medical management for neuropathic pain: a multicentre randomised controlled trial in patients with failed back surgery syndrome. Pain 132(1):179-188

73. Kuncel AM, Grill WM (2004) Selection of stimulus parameters for deep brain stimulation. Clin Neurophysiol 115(11):2431-2441

74. Kuo MF, Paulus W, Nitsche MA (2014) Therapeutic effects of non-invasive brain stimulation with direct currents (tdcs) in neuropsychiatric diseases. Neuroimage 85:948-960

75. Lacour SP, Fitzgerald J, Lago N, Tarte E, McMahon S, Fawcett J (2009) Long micro-channel electrode arrays: A novel type of regenerative peripheral nerve interface. IEEE Trans Neural Syst Rehabil Eng 17(5):454-460

76. Laxpati NG, Mahmoudi B, Gutekunst CA, Newman JP, ZellerTownson R, Gross RE (2014) Real-time in vivo optogenetic neuromodulation and multielectrode electrophysiologic recording with neurorighter. Front Neuroeng 7(40):1-15

77. Legon W, Sato TF, Opitz A, Mueller J, Barbour A, Williams A, Tyler WJ (2014) Transcranial focused ultrasound modulates the activity of primary somatosensory cortex in humans. Nat Neurosci 17(2):322-329

78. Lesser RP, Kim SH, Beyderman L, Miglioretti DL, Webber WRS, Bare M, Cysyk B, Krauss G, Gordon B (1999) Brief bursts of pulse stimulation terminate after discharges caused by cortical stimulation. Neurology 53(9):2073-2073

79. Levy R, Ashby P, Hutchison WD, Lang AE, Lozano AM, Dostrovsky JO (2002) Dependence of subthalamic nucleus oscillations on movement and dopamine in Parkinsons disease. Brain 125(6):1196-1209

80. Liew S, Santarnecchi E, Buch E, Cohen L (2014) Noninvasive brain stimulation in neurorehabilitation: Local and distant effects for motor recovery. Front Human Neurosci 8:378

81. Little S, Brown P (2012) What brain signals are suitable for feedback control of deep brain stimulation in Parkinson's disease? Ann N Y Acad Sci 1265(1):9-24
82. Lucas TH, Fetz EE (2013) Myo-cortical crossed feedback reorganizes primate motor cortex output. J Neurosci 33(12):5261-5274

83. Mavoori J, Jackson A, Diorio C, Fetz E (2005) An autonomous implantable computer for neural recording and stimulation in unrestrained primates. J Neurosci Methods 148(1):71-77

84. Mayberg HS, Lozano AM, Voon V, McNeely HE, Seminowicz D, Hamani C, Schwalb JM, Kennedy SH (2005) Deep brain stimulation for treatment-resistant depression. Neuron 45(5):651-660

85. Moritz CT, Perlmutter SI, Fetz EE (2008) Direct control of paralysed muscles by cortical neurons. Nature 456(7222):639-642

86. Morrell MJ (2011) Responsive cortical stimulation for the treatment of medically intractable partial epilepsy. Neurology 77(13):1295-1304

87. Morris GL, Gloss D, Buchhalter J, Mack KJ, Nickels K, Harden C (2013) Evidence-based guideline update: Vagus nerve stimulation for the treatment of epilepsy report of the guideline development subcommittee of the american academy of neurology. Neurology 81(16):1453-1459

88. Muller R, Gambini S, Rabaey J (2012) A $0.013 \mathrm{~mm}^{2} 5 \mu \mathrm{W}$ DCcoupled neural signal acquisition IC with $0.5 \mathrm{~V}$ supply. IEEE J Solid State Circuits 47(1):232-243

89. Nag S, Thakor NV (2016) Implantable neurotechnologies: electrical stimulation and applications. Med Biol Eng Comput 54(1). doi:10.1007/s11517-015-1442-0

90. Neuropace Inc (2015) RNS System User Manual. http://www. neuropace.com/wp-content/uploads/2015/11/UserManual.pdf

91. Newman JP, Zeller-Townson R, Mf Fong, Arcot Desai S, Gross RE, Potter SM (2013) Closed-loop, multichannel experimentation using the open-source neurorighter electrophysiology platform. Front Neural Circuits 6(98):1-18

92. Ng KA, Greenwald E, Xu YP, Thakor NV (2016) Implantable neurotechnologies: a review of integrated circuit neural amplifiers. Med Biol Eng Comput 54(1). doi:10.1007/s11517-015-1431-3

93. Nishimura Y, Perlmutter SI, Eaton RW, Fetz EE (2013) Spiketiming-dependent plasticity in primate corticospinal connections induced during free behavior. Neuron 80(5):1301-1309

94. Nishimura Y, Perlmutter SI, Fetz EE (2013) Restoration of upper limb movement via artificial corticospinal and musculospinal connections in a monkey with spinal cord injury. Front Neural Circuits 7(57):1-9

95. Nitsche MA, Paulus W (2011) Transcranial direct current stimulation-update 2011. Restor Neurol Neurosci 29(6):463-492

96. Nudo RJ, Wise BM, SiFuentes F, Milliken GW (1996) Neural substrates for the effects of rehabilitative training on motor recovery after ischemic infarct. Science 272(5269):1791-1794

97. Nuttin B, Cosyns P, Demeulemeester H, Gybels J, Meyerson B (1999) Electrical stimulation in anterior limbs of internal capsules in patients with obsessive-compulsive disorder. Lancet 354(9189): 1526

98. Ochoa J, Torebjörk E (1983) Sensations evoked by intraneural microstimulation of single mechanoreceptor units innervating the human hand. J Physiol 342(1):633-654

99. O’Doherty JE, Lebedev MA, Hanson TL, Fitzsimmons NA, Nicolelis MA (2009) A brain-machine interface instructed by direct intracortical microstimulation. Front Integr Neurosci $3(20): 1-10$

100. O'Doherty JE, Lebedev MA, Ifft PJ, Zhuang KZ, Shokur S, Bleuler H, Nicolelis MAL (2011) Active tactile exploration using a brain-machine-brain interface. Nature 479(7372):228-231

101. Opris I, Fuqua JL, Huettl PF, Gerhardt GA, Berger TW, Hampson RE, Deadwyler SA (2012) Closing the loop in primate prefrontal cortex: Inter-laminar processing. Front Neural Circuits 6(88):1-13

102. Parthasarathy AB, Fox DJ, Dunn AK, Weber EL, Richards LM (2010) Laser speckle contrast imaging of cerebral blood flow 
in humans during neurosurgery: a pilot clinical study. J Biomed Opt 15(6):066030-066030

103. Patil AC, Thakor NV (2016) Implantable neurotechnologies: a review of micro and nano-electrodes for neural recording. Med Biol Eng Comput 54(1). doi:10.1007/s11517-015-1430-4

104. Paz JT, Davidson TJ, Frechette ES, Delord B, Parada I, Peng K, Deisseroth K, Huguenard JR (2013) Closed-loop optogenetic control of thalamus as a tool for interrupting seizures after cortical injury. Nat Neurosci 16(1):64-70

105. Plow EB, Carey JR, Nudo RJ, Pascual-Leone A (2009) Invasive cortical stimulation to promote recovery of function after stroke a critical appraisal. Stroke 40(5):1926-1931

106. Polasek KH, Hoyen HA, Keith MW, Kirsch RF, Tyler DJ (2009) Stimulation stability and selectivity of chronically implanted multicontact nerve cuff electrodes in the human upper extremity. IEEE Trans Neural Syst Rehabil Eng 17(5):428-437

107. Pons T, Garraghty P, Ommaya A, Kaas J, Taub E, Mishkin M (1991) Massive cortical reorganization after sensory deafferentation in adult macaques. Science 252(5014):1857-1860

108. Priori A, Foffani G, Pesenti A, Tamma F, Bianchi A, Pellegrini M, Locatelli M, Moxon K, Villani R (2004) Rhythm-specific pharmacological modulation of subthalamic activity in Parkinson's disease. Exp Neurol 189(2):369-379

109. Priori A, Foffani G, Rossi L, Marceglia S (2013) Adaptive deep brain stimulation (aDBS) controlled by local field potential oscillations. Exp Neurol 245:77-86

110. Raspopovic S, Capogrosso M, Petrini FM, Bonizzato M, Rigosa J, Di Pino G, Carpaneto J, Controzzi M, Boretius T, Fernandez E et al (2014) Restoring natural sensory feedback in real-time bidirectional hand prostheses. Sci Transl Med 6(222):222ra19-222ra19

111. Rebesco JM, Stevenson IH, Körding KP, Solla SA, Miller LE (2010) Rewiring neural interactions by micro-stimulation. Front Syst Neurosci 4(39):1-15

112. Reger BD, Fleming KM, Sanguineti V, Alford S, Mussa-Ivaldi FA (2000) Connecting brains to robots: an artificial body for studying the computational properties of neural tissues. Artif Life 6(4):307-324

113. Rhew HG, Jeong J, Fredenburg J, Dodani S, Patil P, Flynn M (2014) A fully self-contained logarithmic closed-loop deep brain stimulation $\mathrm{SoC}$ with wireless telemetry and wireless power management. IEEE J Solid State Circuits 99:1-15

114. Romo R, Hernndez A, Zainos A, Brody CD, Lemus L (2000) Sensing without touching: Psychophysical performance based on cortical microstimulation. Neuron 26(1):273-278

115. Romo R, Hernndez A, Zainos A, Salinas E (1998) Somatosensory discrimination based on cortical microstimulation. Nature 392:387

116. Rosin B, Slovik M, Mitelman R, Rivlin-Etzion M, Haber SN, Israel Z, Vaadia E, Bergman H (2011) Closed-loop deep brain stimulation is superior in ameliorating parkinsonism. Neuron 72(2):370-384

117. Rossini PM, Micera S, Benvenuto A, Carpaneto J, Cavallo G, Citi L, Cipriani C, Denaro L, Denaro V, Pino GD, Ferreri F, Guglielmelli E, Hoffmann KP, Raspopovic S, Rigosa J, Rossini L, Tombini M, Dario P (2010) Double nerve intraneural interface implant on a human amputee for robotic hand control. Clin Neurophysiol 121(5):777-783

118. Rouse AG, Stanslaski SR, Cong P, Jensen RM, Afshar P, Ullestad D, Gupta R, Molnar GF, Moran DW, Denison TJ (2011) A chronic generalized bi-directional brain machine interface. $\mathrm{J}$ Neural Eng 8(3):036018

119. Sainburg RL, Ghilardi MF, Poizner H, Ghez C (1995) Control of limb dynamics in normal subjects and patients without proprioception. J Neurophysiol 73(2):820-835
120. Sasada S, Kato K, Kadowaki S, Groiss SJ, Ugawa Y, Komiyama T, Nishimura Y (2014) Volitional walking via upper limb muscle-controlled stimulation of the lumbar locomotor center in man. J Neurosci 34(33):11131-11142

121. Schiefer MA, Freeberg M, Pinault GJC, Anderson J, Hoyen H, Tyler DJ, Triolo RJ (2013) Selective activation of the human tibial and common peroneal nerves with a flat interface nerve electrode. J Neural Eng 10(5):056006

122. Schwartz AB, Cui XT, Weber DJ, Moran DW (2006) Brain-controlled interfaces: movement restoration with neural prosthetics. Neuron 52(1):205-220

123. Sherman DL, Tsai YC, Rossell LA, Mirski MA, Thakor NV (1997) Spectral analysis of a thalamus-to-cortex seizure pathway. IEEE Trans Biomed Eng 44(8):657-664

124. Silberstein P, Pogosyan A, Kühn AA, Hotton G, Tisch S, Kupsch A, Dowsey-Limousin P, Hariz MI, Brown P (2005) Corticocortical coupling in Parkinson's disease and its modulation by therapy. Brain 128(6):1277-1291

125. Song W, Kerr CC, Lytton WW, Francis JT (2013) Cortical plasticity induced by spike-triggered microstimulation in primate somatosensory cortex. PloS one 8(3):e57453

126. Stanslaski S, Afshar P, Cong P, Giftakis J, Stypulkowski P, Carlson D, Linde D, Ullestad D, Avestruz AT, Denison T (2012) Design and validation of a fully implantable, chronic, closedloop neuromodulation device with concurrent sensing and stimulation. IEEE Trans Neural Syst Rehabil Eng 20(4):410-421

127. Stewart JD (2003) Peripheral nerve fascicles: Anatomy and clinical relevance. Muscle Nerve 28(5):525-541

128. Suminski AJ, Tkach DC, Fagg AH, Hatsopoulos NG (2010) Incorporating feedback from multiple sensory modalities enhances brain machine interface control. J Neurosci 30(50): $16777-16787$

129. Tabot GA, Dammann JF, Berg JA, Tenore FV, Boback JL, Vogelstein RJ, Bensmaia SJ (2013) Restoring the sense of touch with a prosthetic hand through a brain interface. Proc Natl Acad Sci 110(45):18279-18284

130. Tan D, Schiefer M, Keith MW, Anderson R, Tyler DJ (2013) Stability and selectivity of a chronic, multi-contact cuff electrode for sensory stimulation in a human amputee. In: 2013 6th international IEEE/EMBS conference on neural engineering (NER), pp 859-862

131. Tan DW, Schiefer MA, Keith MW, Anderson JR, Tyler J, Tyler DJ (2014) A neural interface provides long-term stable natural touch perception. Sci Transl Med 6(257):257ra138

132. Tessadori J, Bisio M, Martinoia S, Chiappalone M (2012) Modular neuronal assemblies embodied in a closed-loop environment: towards future integration of brains and machines. Front Neural Circuits 6(99):1-16

133. Tyler DJ, Durand DM (2003) Chronic response of the rat sciatic nerve to the flat interface nerve electrode. Ann Biomed Eng 31(6):633-642

134. Vagus Nerve Stimulation Study Group (1995) A randomized controlled trial of chronic vagus nerve stimulation for treatment of medically intractable seizures. Neurology 45:224-230

135. Verma N, Shoeb A, Bohorquez J, Dawson J, Guttag J, Chandrakasan A (2010) A micro-power EEG acquisition SoC with integrated feature extraction processor for a chronic seizure detection system. IEEE J Solid State Circuits 45(4):804-816

136. Volkmann J, Moro E, Pahwa R (2006) Basic algorithms for the programming of deep brain stimulation in Parkinson's disease. Mov Disord 21(S14):S284-S289

137. Weber DJ, Friesen R, Miller LE (2012) Interfacing the somatosensory system to restore touch and proprioception: Essential considerations. J Motor Behav 44(6):403-418 
138. Wise KD, Sodagar AM, Yao Y, Gulari MN, Perlin GE, Najafi K (2008) Microelectrodes, microelectronics, and implantable neural microsystems. Proc IEEE 96(7):1184-1202

139. Yoo J, Yan L, El-Damak D, Altaf M, Shoeb A, Chandrakasan A (2013) An 8-channel scalable EEG acquisition SoC with patientspecific seizure classification and recording processor. IEEE $\mathbf{J}$ Solid State Circuits 48(1):214-228

140. Zanos S, Richardson A, Shupe L, Miles F, Fetz E (2011) The neurochip-2: An autonomous head-fixed computer for recording and stimulating in freely behaving monkeys. IEEE Trans Neural Syst Rehabil Eng 19(4):427-435
141. Zhang F, Mishra A, Richardson A, Otis B (2011) A low-power ECoG/EEG processing IC with integrated multiband energy extractor. IEEE Trans Circuits Systems I Regular Papers 58(9):2069-2082

142. Zhang Y, Zhang F, Shakhsheer Y, Silver J, Klinefelter A, Nagaraju M, Boley J, Pandey J, Shrivastava A, Carlson E, Wood A, Calhoun B, Otis B (2013) A batteryless $19 \mu \mathrm{W}$ MICS/ISM-band energy harvesting body sensor node SoC for ExG applications. IEEE J Solid State Circuits 48(1):199-213 